International Electronic Journal of GeOMEtry

Volume 7 No. 1 PP. 126-132 (2014) (CIEJG

\title{
SYMMETRIC TENSOR RANK, CACTUS RANK AND RELATED COMPLEXITY MEASURES FOR HOMOGENEOUS POLYNOMIALS
}

\author{
E. BALLICO \\ Dedicated to memory of Proffessor Franki Dillen
}

\begin{abstract}
Let $\nu_{d}: \mathbb{P}^{m} \rightarrow \mathbb{P}^{r}, r:=\left(\begin{array}{c}m+d \\ m\end{array}\right)-1$, be the Veronese embedding. For any $P \in \mathbb{P}^{r}$ we define its complexity rank (resp. complexity scheme-rank) as the minimal integer $d_{1}+\cdots+d_{s}$ with $d_{i}$ the degrees of hypersurfaces schemetheoretically cutting a finite set (resp. a zero-dimensional scheme) $Z \subset \mathbb{P}^{m}$ with $P$ in the linear span of $\nu_{d}(Z)$. We study these definitions (and related ones) when either $P$ has border rank $\leq 3$ or $P$ is in the linear span of $\nu_{d}(L)$ for some line $L \subset \mathbb{P}^{m}$
\end{abstract}

For any scheme $Z$ of any projective space $\mathbb{P}^{k}$, let $\langle Z\rangle \subseteq \mathbb{P}^{k}$ denote its linear span, i.e. the intersection of all hyperplanes of $\mathbb{P}^{k}$ containing $Z$, with the convention $\langle Z\rangle=\mathbb{P}^{k}$ if there is no such a hyperplane. For any positive integers $m, d$ let $\nu_{d}: \mathbb{P}^{m} \rightarrow \mathbb{P}^{r}, r:=\left(\begin{array}{c}m+d \\ m\end{array}\right)-1$, denote the order $d$ Veronese embedding of $\mathbb{P}^{m}$. For each $P \in \mathbb{P}^{r}$ the rank $r_{m, d}(P)$ (resp. the cactus rank or scheme-rank $z_{m, d}(P)$ ) of $P$ is the minimal cardinality (resp. minimal degree) of a finite set (resp. a zerodimensional scheme) $Z \subset \mathbb{P}^{m}$ such that $P \in\left\langle\nu_{d}(Z)\right\rangle$ ([12], [11] (where the cactus rank is called the scheme-rank), [5], [4]). The integers $r_{m, d}(P)$ and $z_{m, d}(P)$ are a measure of the complexity of $P$ with respect to homogeneous polynomials. In this note we study other measures of complexity of finite sets and zero-dimensional schemes $Z \subset \mathbb{P}^{m}$. Taking these integers instead of the integer $\operatorname{deg}(Z)$ we get various notions of complexity rank.

For any finite string $\underline{d}=d_{1} \geq \cdots \geq d_{s}$ of positive integers and any positive real number $\alpha$ set $\|\underline{d}\|=\left(\sum_{i=1}^{s} d_{i}^{\alpha}\right)^{1 / \alpha}$. Set $\|\underline{d}\|:=\|\underline{d}\|_{1}$. For each zero-dimensional scheme $Z \subset \mathbb{P}^{m}$ let $c c(Z)$ be the minimal integer $\|\underline{d}\|$, where $d_{1} \geq \cdots \geq d_{s}$ are the degrees of some hypersurfaces $Y_{1}, \ldots, Y_{s}$ cutting out $Z$ scheme-theoretically, i.e. such that $Z=Y_{1} \cap \cdots \cap Y_{s}$ (scheme-theoretic intersection). Let $\widehat{c} r_{m, d}(P)$ (resp. $\left.\widehat{c} z_{m, d}(P)\right)$ be the minimal integer $c c(Z)$ for some finite set (resp. zero-dimensional scheme) $Z$ such that $P \in\left\langle\nu_{d}(Z)\right\rangle$. We say that $\widehat{c} r_{m, d}(P)$ (resp. $\left.\widehat{c} z_{m, d}(P)\right)$ is the complexity rank (resp. complexity scheme-rank) of $P$.

2010 Mathematics Subject Classification. 14N05; 14Q05; 15A69.

Key words and phrases. symmetric tensor rank; cactus rank; complexity rank.

The author was partially supported by MIUR and GNSAGA of INdAM (Italy). 
We also introduce the following two measures of the complexity of the string of integers $\underline{d}$. Set $\|\underline{d}\|_{-}:=\prod_{i=1}^{s} d_{i}$ and $\|\underline{d}\|_{+}:=\prod_{i=1}^{s}\left(d_{i}+1\right)$. The integer $\|\underline{d}\|_{-}$is quite natural (when $s=m$ it would give the degree of a zero-dimensional complete intersection $Z \subset \mathbb{P}^{m}$ cut out by hypersurfaces of degree $\left.d_{1}, \ldots, d_{m}\right)$. The integer $\|\underline{d}\|_{+}$weights more the low degree hypersurfaces. For each zero-dimensional scheme $Z \subset \mathbb{P}^{m}$ let $\check{c} c(Z)$, resp. $\left.\check{c} \check{c}(Z)\right)$ be the minimal integer $\|\underline{d}\|_{-}$(resp. $\|\underline{d}\|_{+}$) where $d_{1} \geq \cdots \geq d_{s}$ are the degrees of some hypersurfaces $Y_{1}, \ldots, Y_{s}$ cutting out $Z$ scheme-theoretically. Let $\check{c} z_{m, d}(P)$ (resp. $\left.\check{c} r_{m, d}(P)\right)$ be the minimal integer $\check{c} c(Z)$ for a zero-dimensional scheme (resp. a finite set) $Z \subset \mathbb{P}^{m}$ such that $P \in\left\langle\nu_{d}(Z)\right\rangle$. Define $\check{c} \check{z}_{m, d}(P)$ and $\check{c} \check{r}_{m, d}(P)$ in the same way using \|\|$_{+}$instead of \|\|$_{-}$.

We work over an algebraically closed field $\mathbb{K}$ with characteristic zero. For the positive characteristic case, see Remark 2.3.

\section{LINEAR SPANS OF RATIONAL NORMAL CURVES}

In this section we first give two preliminary lemmas (Lemma 1.1 and 1.2). Then we shows that schemes evincing either $\widehat{c} z_{m, d}(P)$ or $\check{c} z_{m, d}(P)$ or $\check{c} \check{z}_{m, d}(P)$ for some $P \in \mathbb{P}^{r}, r=\left(\begin{array}{c}m+d \\ m\end{array}\right)-1$, are complete intersection (see Proposition 1.1). Then we consider the case in which, in suitable coordinates, the homogeneous polynomial associated to $P$ is a bivariate polynomial, i.e. the case in which there is a line $L \subset \mathbb{P}^{m}$ such that $P \in\left\langle\nu_{d}(L)\right\rangle$. The curve $\nu_{d}(L)$ is a degree $d$ rational normal curve in its linear span $\left\langle\nu_{d}(L)\right\rangle \subset \mathbb{P}^{r}$ and $\operatorname{dim}\left(\left\langle\nu_{d}(L)\right\rangle\right)=d$.

See [12] or [3] for the notion of border $\operatorname{rank} b_{m, d}(P)$ of any $P \in \mathbb{P}^{r}, r:=\left(\begin{array}{c}m+d \\ m\end{array}\right)-1$, with respect to the Veronese variety $\nu_{d}\left(\mathbb{P}^{m}\right)$.

Lemma 1.1. Let $X$ be an integral projective variety. Fix $L \in \operatorname{Pic}(X)$ and linear subspaces $V \subset W \subsetneq H^{0}(X, L)$. Call $B_{1}, \ldots, B_{x}$ the irreducible components of the set-theoretic base locus of $V$. Assume that none of them is an irreducible component of the set-theoretic base locus of $W$. Fix a general $f \in W$. Then the hypersurface $\{f=0\}$ contains no $B_{i}$ and hence the base locus of $W$ is either empty or with dimension $\leq \max \left\{\operatorname{dim}\left(B_{i}\right)\right\}-1$.

Proof. Use that $W$ is an irreducible variety (and hence that a finite intersection of non-empty open subsets of $W$ is non-empty) and that $x$ is a finite integer.

Lemma 1.2. Let $Z \subset \mathbb{P}^{m}, Z \neq \emptyset$, be a zero-dimensional scheme. Let $a_{1} \geq \cdots \geq$ $a_{s}>0$ be the degrees of a set of polynomials $g_{1}, \ldots, g_{s}$ defining scheme-theoretically $Z$. Set $b_{i}:=a_{i}$ for $1 \leq i \leq m-1$ and $b_{m}:=a_{s}$. Fix a general $f_{i} \in H^{0}\left(\mathcal{I}_{Z}\left(b_{i}\right)\right)$. Then the scheme $\left\{f_{1}=\cdots=f_{m}=0\right\}$ has dimension zero.

Proof. Since $Z \neq \emptyset$, we have $s \geq m$ and hence the integers $b_{1}, \ldots, b_{m}$ are welldefined. For each integer $i \in\{2, \ldots, m\}$ set $U_{j}:=\left\{g_{s}=\cdots=g_{i}=0\right\}$. Let $A(k)$, $1 \leq i \leq m$, the statement that $\left\{f_{m}=\cdots=f_{m-k+1}=0\right\}$ has dimension $m-k$. The lemma is true if $A(m)$ is true. $A(1)$ is true, because $g_{s} \neq 0$ and hence $f_{m} \neq 0$. Fix an integer $k \in\{2, \ldots, m\}$ and assume $A(k-1)$. Let $B_{1}, \ldots, B_{x}$ the irreducible components of the scheme $\left\{f_{m}=\cdots=f_{m-k+2}=0\right\}$. Since $A(k-1)$ is assumed to be true, we have $\operatorname{dim}\left(B_{j}\right)=m-k+1$ for all $i$. By Lemma 1.1 to prove $A(k)$ it is sufficient to see that the base locus $B$ of the linear subspace of $H^{0}\left(\mathcal{I}_{Z}\left(b_{m-k+1}\right)\right)$ spanned by $f_{m}, \ldots, f_{m-k+1}$ has dimension at most $m-k$. Assume that this is not true and take an irreducible component $T$ of $B$ with dimension $>m-k$. We have $T=B_{j}$ for some $j$. Since $Z=\left\{g_{1}=\cdots=g_{s}\right\}$, we have $\operatorname{dim}\left(U_{k}\right) \leq m-k$. Since 
$U_{k}$ is contained in the base locus $\Delta$ of $\left|\mathcal{I}_{Z}\left(b_{k}\right)\right|, \Delta$ has dimension at most $k$. Hence $f \mid T \neq 0$ for a general $f \in H^{0}\left(\mathcal{I}_{Z}\left(b_{k}\right)\right.$, a contradiction.

Proposition 1.1. Fix positive integers $m, d$, any $P \in \mathbb{P}^{r}, r:=\left(\begin{array}{c}m+d \\ m\end{array}\right)-1$, and any zero-dimensional scheme $Z \subset \mathbb{P}^{m}$ evincing either $\widehat{c} z_{m, d}(P)$ or $\check{c} z_{m, d}(P)$ or $\check{c} \check{z}_{m, d}(P)$. Then $Z$ is a complete intersection.

Proof. Let $a_{1} \geq \cdots \geq a_{s}$ be the degrees of a minimal set of generators of the homogeneous ideal of $Z$. We have $s \geq m$ and $s=m$ if and only if $Z$ is a complete intersection. We have $c c(Z)=d_{1}+\cdots+d_{s}$. By Lemma 1.2 there is a zerodimensional scheme $W$ containing $Z$ and with $c c(W)=d_{s}+\sum_{i=1}^{m-1} d_{i}$. Since $W \supseteq Z$ we have $P \in\left\langle\nu_{d}(W)\right\rangle$. Hence $c c(Z)=\widehat{c} z_{m, d}(P) \leq c c(W)$, i.e. $m=s$.

The same proof works for $\check{c} z_{m, d}(P)$ and $\check{c} \check{z}_{m, d}(P)$.

Theorem 1.1. Fix $P \in \mathbb{P}^{r}, r:=\left(\begin{array}{c}m+d \\ m\end{array}\right)-1$, for some $m \geq 1, d \geq 3$. Assume the existence of a line $L$ such that $P \in\left\langle\nu_{d}(L)\right\rangle$. Then $\widehat{c} z_{m, d}(P)=z_{m, d}(P)+m-1$ and $\widehat{c} r_{m, d}(P)=r_{m, d}(P)+m-1$.

Proof. Set $b:=z_{m, d}(P)$. By a theorem of Sylvester the integer $b$ is the border rank of $P$ with respect to the rational normal curve $\nu_{d}(L)\left([12]\right.$, citebgi). Let $Z \subset \mathbb{P}^{m}$ be any scheme evincing $z_{m, d}(P)$ and $A \subset \mathbb{P}^{m}$ any scheme evincing $r_{m, d}(P)$. We have $Z \subset L$ and $A \subset L$ ([12], Exercise 3.2.2.2, (for $A$ ) and [8], Proposition 2.1 and Corollary 2.2), $Z$ is unique ([11], 1.36 and 1.38, [7], Theorem 1.18, or use [3], Lemma 34) and either $A=Z$ and $r_{m, d}(P)=b$ or $b<(d+2) / 2, r_{m, d}(P)=d+2-b$ and $A \cap Z=\emptyset$. Since $L$ is the complete intersection of $m-1$ hyperplane and $Z$ (resp. $A) A$ are the complete intersection of $L$ and a hypersurface of degree $\operatorname{deg}(Z)$, resp, $\operatorname{deg}(A))$, we have $c c(Z) \leq b+m-1$ and $c c(A) \leq r_{m, d}(P)+m-1$. Hence $\widehat{c} z_{m, d}(P) \leq b+m-1$ and $\widehat{c} r_{m, d}(P) \leq r_{m, d}(P)+m-1$. Therefore it is sufficient to prove the inequalities the opposite inequalities.

Let $W \subset \mathbb{P}^{m}$ be any zero-dimensional scheme evincing $\widehat{c} z_{m, d}(P)$. By Proposition $1.1 W$ is a complete intersection, say of forms of degree $d_{1} \geq \cdots \geq d_{m}$. To prove that $\widehat{c} z_{m, d}(P)=b+m-1$ it is sufficient to prove that $d_{1}+\cdots+d_{m} \geq b+m-1$. Assume $d_{1}+\cdots+d_{m} \leq b+m-2$.

First assume $W \supseteq Z$. Since $W$ is zero-dimensional, the scheme $W \cap L$ is a zero-dimensional scheme containing $Z$. Hence $e:=\operatorname{deg}(W \cap L) \geq b$. Since at least one of the forms, $F_{i}$, does not vanish identically on all $L$, we have $d_{1} \geq b$. Hence $d_{1}+\cdots+b_{m} \geq b+m-1$.

Now assume $W \nsupseteq Z$. In this case the proof of [2], Lemma 1, gives $h^{1}\left(\mathcal{I}_{Z \cup W}(d)\right)>$ 0 . Since $W$ is a complete intersection, we have $h^{1}\left(\mathcal{I}_{W}\left(d_{1}+\cdots+d_{m}-m-1\right)\right)=1$. Since $\operatorname{deg}(Z)=b$, we have $h^{1}\left(\mathcal{I}_{Z \cup W}\left(d_{1}+\cdots+d_{m}-m-1\right)\right) \leq 1+b$. For any zero-dimensional scheme $B \subset \mathbb{P}^{m}$ the map $\mathbb{N} \rightarrow \mathbb{N}$ defined by $t \mapsto h^{1}\left(\mathcal{I}_{B}(t)\right)$ is strictly decreasing, until it is zero (e.g., because its different function is the Hilbert function of a graded Artinian ring). Hence $h^{1}\left(\mathcal{I}_{Z \cup W}\left(d_{1}+\cdots+d_{m}-m-1+b\right)\right)=0$. Therefore $d \leq-1+d_{1}+\cdots+d_{m}-m-1+b$, i.e. $d_{1}+\cdots+d_{m} \geq d-b+m-2$. Since $2 b \leq d+2$, we get $c c(W) \geq b+m$ in this case.

Now assume $r_{m, d}(P) \neq b_{r, m}(P)$ and hence $r_{m, d}(P)=d+2-b$. Take any $S \subset \mathbb{P}^{m}$ evincing $\widehat{c} r_{m, d}(P)$ and let $a_{1} \geq \cdots \geq a_{s}$ be the sequence of degrees of forms with $S$ as their scheme-locus and with $a_{1}+\cdots+a_{s}$ minimal. Assume $a_{1}+\cdots+a_{s}=\widehat{c} r_{m, d}(P) \leq d+m-b$. Let $N \supseteq S$ be the zero-locus of general $g_{i} \in\left|\mathcal{I}_{Z}\left(b_{i}\right)\right|$ with $b_{m}=d_{s}$ and $b_{i}=a_{i}$ for all $i \in\{1, \ldots, m-1\}$. Lemma 1.2 gives 
$\operatorname{dim}(N)=0$. Since $N \supseteq S$, we have $P \in\left\langle\nu_{d}(N)\right\rangle$. First assume $Z \nsupseteq N$. Since $P \in\left\langle\nu_{d}(Z)\right\rangle \cap\left\langle\nu_{d}(N)\right\rangle$, the proof of [2], Lemma 1, gives $h^{1}\left(\mathcal{I}_{N \cup Z}(d)\right)>0$. Since $N$ is a complete intersection, we have $h^{1}\left(\mathcal{I}_{N}\left(b_{1}+\cdots+b_{m}-m-1\right)\right)=1$. Since $\operatorname{deg}(Z)=b$, we get $h^{1}\left(\mathcal{I}_{Z \cup N}\left(b_{1}+\cdots+b_{m}-m-1\right)\right) \leq 1+b$. As above we get $h^{1}\left(\mathcal{I}_{Z \cup N}\left(b_{1}+\cdots+b_{m}-m-1+b+1\right)\right)=0$. Hence $d \leq b_{1}+\cdots+b_{m}-m-1+b$. Since $b_{1}+\cdots+b_{m} \leq a_{1}+\cdots+a_{s} \leq d+m-b$, we get a contradiction.

Now assume $Z \subseteq N$. Since $S$ is reduced, $Z$ is not reduced and $S$ is schemetheoretically cut-out by forms of degree $a_{1}$, there is $F \in\left|\mathcal{I}_{S}\left(a_{1}\right)\right|$ such that $F \mid Z \neq 0$. Hence for a general $G \in\left|\mathcal{I}_{S}\left(a_{1}\right)\right|$ we have $G \mid Z \neq 0$. The proof that $A(m-1)$ implies $A(m)$ in the proof of Lemma 1.2 gives $N \nsupseteq Z$, a contradiction.

Corollary 1.1. Fix $P \in \mathbb{P}^{r}, r:=\left(\begin{array}{c}m+d \\ m\end{array}\right)-1$, with border rank 2. Then $\operatorname{ccr}_{m, d}(P)=$ $r_{m, d}(P)+m-1$ and $c c z_{m, d}(P)=m+1$.

Proof. E.g., by the proof of [3], Theorem 32, or by [6], $\S 2.1, P \in\langle L\rangle$ for some line $L \subset \mathbb{P}^{m}$. Apply Theorem .

\section{BORDER RANK 3}

Lemma 2.1. Fix a line $L \subset \mathbb{P}^{m}$, a zero-dimensional scheme $W \subset L$ with $e:=$ $\operatorname{deg}(W) \geq 2$ and $O \in \mathbb{P}^{m} \backslash L$. Then the homogeneous ideal $I_{A}$ of $A:=W \cup\{O\}$ is generated by the equation of a degree e cone $F$ with $F \cap L=W$ and $(m-2)$ dimensional vertex containing $O$ and by two degree reducible quadrics formed by the union of two hyperplanes, one containing $L$ and the other one containing $O$, but not $L$ and (if $m>2$ ) the $m-2$ linear equations of the plane $\langle L \cup\{O\}\rangle$.

Proof. It is sufficient to do the case $m=2$. Let $G \subset \mathbb{P}^{2}$ be any hypersurface containing $A$, but not $L$. Then $G \cap L \supseteq W$ and hence $\operatorname{deg}(G) \geq e$ with equality if and only if $G \cap L=W$. We get that the degree two part of $I_{A}$ is generated by the equations of two pairs of line through $O$ and (if $e=2$ ) the equation of $F$. We also get that no generators of $I_{A}$ occurs in degree $<e$. It is easy to check that $h^{1}\left(\mathcal{I}_{A}(e-1)\right)=0$ (even if $e=2$ ). Hence the Castelnuovo-Mumford lemma gives that $I_{A}$ is generated in degree $\leq e$. Since any two degree $e$ elements of $I_{A}$ not containing $L$ induce the same degree $e$ divisor on $L$, we get that $I_{A}$ is minimally generated by the two reducible conics through $O$ containing $L$ and by the equation of $F$.

Theorem 2.1. Fix $P \in \mathbb{P}^{r}, r:=\left(\begin{array}{c}m+d \\ m\end{array}\right)-1, m \geq 2$, with border rank 3. Assume $d \geq 7$.

(a) If there is a line $L \subset \mathbb{P}^{m}$ such that $P \in\left\langle\nu_{d}(L)\right\rangle$, then $\widehat{c} c r_{m, d}(P)=$ $r_{m, d}(P)+m-1$ and $\widehat{c} c z_{m, d}(P)=m+1$.

(b) Assume that there is no line $L \subset \mathbb{P}^{m}$ such that $P \in\left\langle\nu_{d}(L)\right\rangle$.

(b1) We have $\widehat{c} c z_{m, d}(P)=m+4$.

(b2) If $r_{m, d}(P)=3$, then $\widehat{c} c r_{m, d}(P)=m+4$.

(b3) If $r_{m, d}(P)=d+1$, then $\widehat{c} c r_{m, d}(P)=m+4$.

(b4) In all other cases (i.e. if $r_{m, d}(P)=2 d-1$ ), then $\widehat{c} c r_{m, d}(P)=2 d+m$.

Proof. By the proof of [3], Theorem 37, we have $r_{m, d}(P)=2 d-1$ if and only if $P$ is neither as in (a) nor as in (b2) nor as in (b3).

Part (a) is true by Corollary 1.1.

In the set-up of part (b) we fix a scheme $Z$ evincing $z_{m, d}(P)$ (and hence with degree 3 by [6], Proposition 1.2) and a set $S \subset \mathbb{P}^{m}$ evincing $r_{m, d}(P) . Z$ spans a 
plane, because there is no line $L \subset \mathbb{P}^{m}$ such that $P \in\left\langle\nu_{d}(L)\right\rangle$. In this case $\langle Z\rangle$ is a plane. We also know that $Z$ is either a union of 3 non-collinear points, or a connected curvilinear scheme or $Z=v \sqcup\{O\}$ with $v$ connected, $\operatorname{deg}(v)=2$ and $O \notin\langle v\rangle$. In all cases $Z$ is contained in a smooth conic $C$. It is easy to check first that $Z$ is scheme-theoretically cut out in $C$ by two conics and then that the homogeneous ideal of $Z$ is generated by 3 quadratic equations and (if $m>2$ ) the $m-2$ linear equations of $\langle Z\rangle$. Hence $c c z_{m, d}(P) \leq 6+m-2=m+4$. The opposite inequality is obvious, because $Z$ is neither a complete intersection nor contained in a line. We get parts (b1) and (b2). Now assume $r_{m, d}(P)=d+1$. This is the case if and only if $Z=v \sqcup\{O\}$ with $v$ connected, $\operatorname{deg}(v)=2$ and $O \notin\langle v\rangle$ ([3], proof of Theorem 32). Fix any $S$ evincing $r_{m, d}(P)$. By [1], Theorem 4, we have $S=S^{\prime} \sqcup\{O\}$ with $O \notin\langle v\rangle$. The case $e=d$ of Lemma 2.1 gives $c c r_{m, d}(P)=m+4$.

Now assume $r_{m, d}(P)=2 d-1$, i.e. assume that $Z$ is connected and not contained in a line. In this case $Z$ contained in a smooth conic. Fix any $S \subset \mathbb{P}^{m}$ evincing $r_{m, d}(P)$. By [1], Theorem 4, we have $S \cap Z=\emptyset$ and $S \cup Z$ is contained in a reduced conic $T$. The homogeneous ideal of $S$ has exactly $m-2$ linearly independent linear forms. First assume that $T$ is smooth. In this case $S$ is the scheme-theoretic intersection of two degree $2 d$ divisors of $T$, because $T \cong \mathbb{P}^{1}$. Since $T$ is arithmetically Cohen-Macaulay, each of these divisors is the intersection of $T$ with a degree $d$ hypersurface. We get that $S$ is scheme-theoretically cut out by $m-2$ linear forms, a quadratic form and two degree $d$ forms. Hence $\widehat{c} r_{m, d}(P) \leq 2 d+m$. Bezout theorem gives that every $Y \in\left|\mathcal{I}_{S}(d-1)\right|$ contains $T$. Hence we get that in any set of forms defining scheme-theoretically $S$, at least two of these forms have degree at least $d$. Since $S$ is not a complete intersection, to define scheme-theoretically $S$ we need at least $m+1$ forms. Hence $\widehat{c} c r_{m, d}(P) \geq 2 d+m$. Now assume that $T$ is not smooth. Since $S$ is reduced, $T$ is reduced and, calling $L, R$ the components of $T$ with $\sharp(L \cap S) \geq \sharp(R \cap S)$, and $O=L \cap R$ the singular point of $T$, we have $O \notin S,\{O\}=Z_{\text {red }}, \sharp(L \cap S)=d$ and $\sharp(S \cap L)=d-1$ ([1], part (f) of $\left.\S 4\right)$. Since $O \notin S$, there are two plane degree $d$ curves $T_{1}, T_{2}$ in $\langle T\rangle$ such that $T \cap T_{1} \cap T_{2}=S$ (as schemes); we may take as each $T_{i}$ a union of $d$ lines each of them spanned by a point of $S \cap L$ and a point of $S \cap R$.

Remark 2.1. Take $P$ as in Theorem 2.1. Assume $r_{m, d}(P)>3$. By the proof of [3], Theorem 37, there is a line $L \subset \mathbb{P}^{m}$ such that $P \in\left\langle\nu_{d}(L)\right\rangle$ if and if $r_{m, d}(P)=d-1$. In all cases the proof of Theorem 2.1 gives that all sets evincing $r_{m, d}(P)$ have the same complexity.

Remark 2.2. Assume char $(\mathbb{K})=0$. Fix a finite set $S \subset \mathbb{P}^{k}, k \geq 1$, and an integer $s \geq 1$ such that the sheaf $\mathcal{I}_{S}(x)$ is spanned. Then there are $k$ hypersurfaces $F_{i} \in \mid \mathcal{I}_{S}(x)$ such that the scheme $F_{1}=\cdots=F_{k}=0$ is a reduced union of $x^{k}$ points.

Lemma 2.2. Fix $S \subset \mathbb{P}^{s}$ with $\sharp(S)=s+1$ and $\langle S\rangle$. Fix any integer $x \geq 2$. Let $A \subset \mathbb{P}^{s}$ be the intersection of $s$ general elements of $\left|\mathcal{I}_{S}(x)\right|$. Then $A$ is a reduced zero-dimensional scheme with cardinality $x^{s}$.

Proof. Any two such sets are projectively normal. Hence it is sufficient to note that $h^{0}\left(\mathcal{I}_{S}(x)\right)=\left(\begin{array}{c}s+x \\ x\end{array}\right)-s-1 \geq s$ and that a general complete intersection of $s$ hypersurfaces of degree $x$ is smooth ([10], Theorem II.8.12). 
Proposition 2.1. Take a finite set $S \subset \mathbb{P}^{m}, m \geq 1$, which is linearly independent. Set $s:=\sharp(S)$. Fix any $P \in\left\langle\nu_{d}(S)\right\rangle$ such that $P \notin\left\langle\nu_{d}\left(S^{\prime}\right)\right\rangle$ for any $S^{\prime} \subsetneq S$. If $s \leq 2$, then $c c(S)=\widehat{c r} r_{m, d}(P)=\widehat{c} z_{m, d}(P)=s+m-1$. If $s \geq 3$, then $c c(S)=m+s+1$ and $\widehat{c} r_{m, d}(P)=\widehat{c} z_{m, d}(P)=s+m-1$.

Proof. We have $s \leq m+1$. If $s=1,2$, then $S$ is a complete intersection. We have $c c(S)=m+s-1$ (the case $s=1$ is trivial, the case $s=2$ by Corollary 1.1). Now assume $s \geq 3$. Since $\mathcal{I}_{S}(2) \mid$ is spanned and $\operatorname{dim}(\langle S\rangle)=m+1-s, S$ is the scheme-theoretic intersection of $m+1-s$ linearly independent linear forms and some degree two linear forms. Since $S$ is not a complete intersection, Lemma 2.2 gives $c c(S)=(m+1-s)+2 s=m+s+1$. Lemma 2.2 gives the existence of a reduced set $A \supset S$ which is the complete intersection of $m+1-s$ linear forms and $s-1$ degree two forms. Take $B \subset \mathbb{P}^{m}$ evincing $\widehat{c} z_{m, d}(P)$. Since $P$ depends exactly on $s$ homogeneous coordinate and $P \in\left\langle\nu_{d}(B)\right\rangle$, we have $\operatorname{dim}(\langle B\rangle) \geq s-1$. Hence $d_{i}>1$ for all $i \leq \operatorname{dim}(\langle B\rangle)$. Hence $c c(B) \geq m+s-1$.

In the same way we get the following result.

Proposition 2.2. Fix $m \geq 2$ and take any linearly independent zero-dimensional scheme $Z \subset \mathbb{P}^{m}$. Set $s:=\operatorname{deg}(Z)$. Fix any $P \in\left\langle\nu_{d}(Z)\right\rangle$ such that $P \notin\left\langle\nu_{d}\left(Z^{\prime}\right)\right\rangle$ for any $Z^{\prime} \subsetneq Z$. If $s \leq 2$, then $c c(Z)=\widehat{c} z_{m, d}(P)=s+m-1$. If $s \geq 3$, then $c c(Z)=m+s+1$ and $\widehat{c} z_{m, d}(P)=s+m-1$.

Proposition 2.3. Fix $P \in \mathbb{P}^{r}, r:=\left(\begin{array}{c}m+d \\ m\end{array}\right)-1$, for some $m \geq 1, d \geq 3$. Assume the existence of a line $L$ such that $P \in\left\langle\nu_{d}(L)\right\rangle$. Then $\check{c} z_{m, d}(P)=z_{m, d}(P)$, $\check{r} r_{m, d}(P)=$ $r_{m, d}(P), \check{c} r_{m, d}(P)=r_{m, d}(P)=2^{m-1}\left(z_{m, d}(P)+1\right)$ and $\check{c} \check{r}_{m, d}(P)=2^{m-1}\left(r_{m, d}(P)+\right.$ 1).

Proof. Set $b:=z_{m, d}(P)$ and take $Z$ evincing $z_{m, d}(P)$. Recall that $Z \subset L$ ([8], Proposition 2.1 and Corollary 2.2). Hence $Z$ is a complete intersection of a degree $b$ hypersurface and $m-1$ hyperplane. Hence $\|Z\|_{-}=b$ and $\|Z\|_{+}=2^{m-1}(b+1)$. Since for any zero-dimensional scheme $W \subset \mathbb{P}^{m}$ we have $\operatorname{deg}(W) \leq\|W\|_{-}$(with equality if and only if $W$ is a complete intersection), then we get $\check{c} z_{m, d}(P)=b$ and that $Z$ is the only scheme evincing $\check{c} z_{m, d}(P)$. If $r_{m, d}(P)=z_{m, d}(P)$, i.e. if $Z$ is reduced, then we also get $\check{c} r_{m, d}(P)=b$. Now assume $r_{m, d}(P) \neq z_{m, d}(P)$. In this case $2 b \leq d+1, r_{m, d}(P)=d+2-b$ and every set, $A$, evincing $r_{m, d}(P)$ is contained in $A$. The set $A$ is the complete intersection of $m-1$ hyperplanes and a degree $d+2-b$ hypersurface and hence $\check{c} c(A)=d+2-b$. Since any $A$ evincing $r_{m, d}(P)$ is contained in $L$ ([12], Exercise 3.2.2.2, or [8], Proposition 2.1 and Corollary 2.2), we get $\check{c} r_{m, d}(P)=r_{m, d}(P)$..

Remark 2.3. Assume $p:=\operatorname{char}(\mathbb{K})>0$. It is sufficient to ass assume $p>d$, because Sylvester theorem quoted and proved in characteristic zero in [9] and [3] is true in positive characteristic if $p>d$ ([11], page 22).

\section{REFERENCES}

[1] Ballico, E., Subsets of the variety $X \subset \mathbb{P}^{n}$ evincing the $X$-rank of a point of $\mathbb{P}^{n}$, preprint.

[2] Ballico, E. and Bernardi, A., Decomposition of homogeneous polynomials with low rank, Math. Z. 271 (2012) 1141-1149.

[3] Bernardi, A., Gimigliano, A. and Idà, M., Computing symmetric rank for symmetric tensors, J. Symbolic. Comput. 46 (2011), no. 1, 34-53.

[4] Bernardi, A. and Ranestad, K., The cactus rank of cubic forms, J. Symbolic. Comput. 50 (2013) 291-297. DOI: 10.1016/j.jsc.2012.08.001 
[5] Buczyńska, W. and Buczyński, J., Secant varieties to high degree veronese reembeddings, catalecticant matrices and smoothable Gorenstein schemes. arXiv:1012.3562v4 [math.AG], J. Algebraic Geom. (to appear).

[6] Buczyńska, W. and Buczyński, J., On the difference between the border rank and the smoothable rank of a polynomial, arXiv:1305.1726.

[7] Buczyński, J., Ginensky, A. and Landsberg, J. M., Determinantal equations for secant varieties and the Eisenbud-Koh-Stillman conjecture, J. London Math. Soc. (2) 88 (2013) 1-24.

[8] Buczyński, J. and Landsberg, J. M., Ranks of tensors and a generalization of secant varieties, Linear Algebra Appl. 438 (2013), no. 2, 668-689.

[9] Comas, G., and Seiguer, M., On the rank of a binary form, Found. Comp. Math. 11 (2011), no. $1,65-78$.

[10] Hartshorne, R., Algebraic Geometry, Springer, Berlin, 1977.

[11] Iarrobino, A. and Kanev.,V., Power sums, Gorenstein algebras, and determinantal loci. Lecture Notes in Mathematics, vol. 1721, Springer-Verlag, Berlin, 1999, Appendix C by Iarrobino and Steven L. Kleiman.

[12] Landsberg, J. M., Tensors: Geometry and Applications, Graduate Studies in Mathematics, Vol. 128, Amer. Math. Soc. Providence, 2012.

Dept. of Mathematics, University of Trento, 38123 Povo (TN), Italy

E-mail address: ballico@science.unitn.it 